\title{
Tension Pneumomediastinum in a Patient with Interstitial Pneumonia
}

\author{
Takayuki Takimoto, Satomu Morita, Akio Osa and Kinya Abe
}

Key words: tension pneumomediastinum, interstitial pneumonia, pneumothorax

(Intern Med 51: 125, 2012)

(DOI: 10.2169/internalmedicine.51.6515)

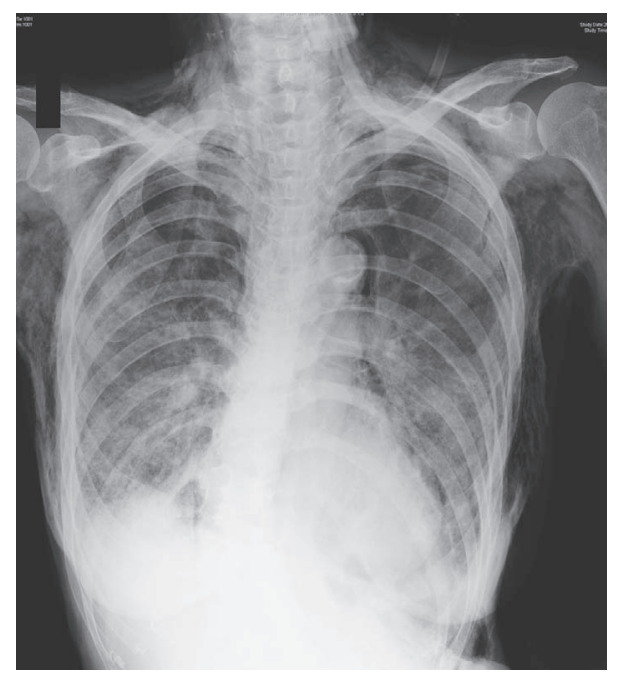

Picture 1A.

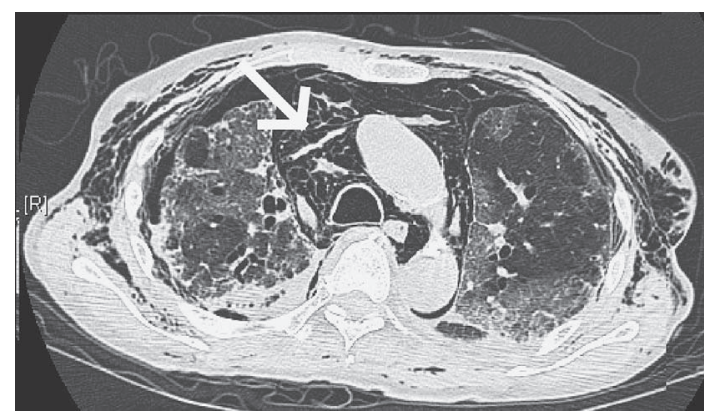

Picture 1B.

A 72-year-old woman was diagnosed as acute exacerba- tion of interstitial pneumonia (IP) on admission. She received steroid therapy, but, her respiratory condition deteriorated progressively. Chest radiograph (Picture 1A) and thoracic computed tomography (Picture $1 \mathrm{~B}$ ) on hospital day 22 demonstrated severe pneumomediastinum, bilateral pneumothorax and subcutaneous emphysema, in addition to diffuse interstitial opacities. The superior vena cava was severely compressed by mediastinal air (1B, arrow). Drainage of the right thoracic cavity was not effective, and she died on hospital day 28 .

Tension pneumomediastinum is described as a serious condition, leading to cardiac compression by mediastinal air. In IP, extra-alveolar air is recognized as a relatively common complication $(1,2)$; however, tension pneumomediastinum is rare. Pneumomediastinum is suggested to be due to the rupture of alveoli or honeycomb cysts secondary to $\operatorname{IP}(1,2)$, and to further deteriorate by steroid therapy. Prompt decompression, by mediastinal tube placement or mediastinotomy, should be considered.

The authors state that they have no Conflict of Interest (COI).

\section{References}

1. Macklin MT, Macklin CC. Malignant interstitial emphysema of the lungs and mediastinum as an important occult complication in many respiratory diseases and other conditions: an interpretation of the clinical literature in the light of laboratory experiment. Medicine 23: 281-358, 1944.

2. Fujiwara T. Pneumomediastinum in pulmonary fibrosis. Detection by computed tomography. Chest 104: 44-46, 1993.

Department of Internal Medicine, Toyonaka Municipal Hospital, Japan

Received for publication September 8, 2011; Accepted for publication October 11, 2011

Correspondence to Dr. Takayuki Takimoto, taka2003@chp.toyonaka.osaka.jp

(C) 2012 The Japanese Society of Internal Medicine Journal Website: http://www.naika.or.jp/imindex.html 